\title{
Numerical Construction of Nonlinear Optical Evolution of Femtosecond Laser Pulse Propagation in Air Based on 2D+1 NLSE Full Model
}

\author{
Yuheng Wang ${ }^{1 *}$, Olivier Uteza ${ }^{2}$, Qingbang Shu ${ }^{1}$, Zhenwei Cui ${ }^{1}$, Zilong Cong ${ }^{1}$, Marc Sentis ${ }^{2}$ \\ ${ }^{1}$ The Center for Numerical Simulation and Software, Northwest Institute of Nuclear \\ Technology(NINT), 710024 Xi'an, China \\ *E-mail: imtree@126.com \\ ${ }^{2}$ Aix Marseille Univ., CNRS, LP3, Marseille, France
}

\begin{abstract}
We develop a tool for numerical construction of femtosecond laser pulse propagation in air. The model is based on 2D+1 nonlinear Schrödinger equation (NLSE). Several nonlinear physics effects are considered in this full model which includes High-order Kerr effect (HOKE), Raman Kerr contribution, plasma absorption and defocusing. It is often implemented in laboratory with weak focusing optics for better observation, and the practical applications always involve collimated or nearly collimated beams. Here, we carry out a series of simulation on propagation of collimated femtosecond laser pulse in air, with peak power around the critical power for self-focusing $\left(P_{\mathrm{cr}}\right)$. The results are shown for peak power respectively equal to 0.3 and $1.9 P_{\text {cr. Especially, we numerically construct }}$ a complex physical processes image involving filamentation which is regarded as a delicate task to interpret numerical results with many competing effects. The results show a dynamic equilibrium between Kerr self-focusing and HOKE in case of $1.9 P_{\mathrm{cr}}$. HOKE shows dominant in the competition of nonlinear effects so as to balance Kerr self-focusing, at least for the pulse with $100 \mathrm{fs}$ at $800 \mathrm{~nm}$. Our analysis is in accordance with the Marburger's prediction and image found in published literatures. Perspective of these works will be dedicated to describe the propagation of ultrashort pulses (around $15 \mathrm{fs}$ ) with NA $~ 0.1$ in the context of laser ablation in order to shed light on the significance of air on the outcomes of the laser-matter interaction.
\end{abstract}

DOI: 10.2961/jlmn.2017.01.0006

Keywords: nonlinear propagation, femtosecond laser pulse, filamentation, self-focusing, Highorder Kerr effect

\section{Introduction}

The propagation of femtosecond laser pulses attracts much interest due to its spectacular potential application. The objective of the working program is to establish a precise and reliable numerical tool able to describe the propagation of femtosecond pulse of variable duration ( $10 \mathrm{fs}-$ $500 \mathrm{fs}$ ), in air and at atmospheric pressure and $20^{\circ} \mathrm{C}$ temperature. The simulations of the propagation of a femtosecond pulse in air developed by our joined group have a strong interest for the damage and ablation experiments, corresponding to the experimental laser sources available in laboratory ${ }^{[1-3]}$. Indeed, they would help to define with high precision the exact distribution of intensity on the target that can be further compared to experiments ${ }^{[1-5]}$, and to shed light on the significance of air on the outcomes of the laser-matter interaction. Those developments are particularly important in the context of laser damage measurements of optical materials and components ${ }^{[2,3]}$, a crucial topic for the development of high peak power PW lasers, and also for the development of high resolution and high control micromachining processes ${ }^{[4,5]}$ for further potential applications in photonics industry.

It is often considered as a typical linear process when the continuous wave and long pulse laser transmit in vacuum. The diffraction and dispersion effects could be regarded as independent without mutual coupling. However, there are some challenges in research of the propagation of femtosecond pulse, which shows special performance due to its ultrashort pulse duration and spatial-time coupling feature. And it also presents new physical phenomena which is highly nonlinear under the non-vacuum dielectric conditions. The spatial-time coupling effect cannot be ignored, even in vacuum, in the case of the propagation of ultrafast and ultra-strong femtosecond laser pulse. So the theoretical description and analogue simulation on various nonlinear physical effects present great difficulty in such tiny time scale. Meanwhile, effective experimental measurement of various nonlinear physical effects and diagnosis of physical parameters are big challenges. For instance, the power density along the propagation path of pulse cannot be measured directly due to a very high intensity $\left(>10^{13} \mathrm{~W} / \mathrm{cm}^{2}\right)$. It is also extremely difficult to satisfy the experimental measurement requirements of ultrashort pulse characteristics with high resolution in spatial-time domain. Up till now, numerical simulation is an important way to investigate the process of propagation. In general, managing the controllable propagation of the femtosecond laser means to make the laser reaching a predetermined position with the expected accuracy, which has a great significance in applications.

At present, great progresses have been made on the understanding of femtosecond pulse propagation in the last decade, but some of the physical phenomena cannot be explained yet. Propagation in the dispersion medium is 
considered to be a dynamic equilibrium of kinetic process. It has long been believed that one is the self-focusing caused by Kerr effect and the other is the defocusing caused by plasma response ${ }^{[6]}$. These two highly nonlinear effects will induce self-guide propagation over several Rayleigh lengths, with extremely high power density. However, the high order nonlinear effect (HOKE), which will produce a great effect on propagation, was recently considered as a dominant factor in the filamentation regime ${ }^{[7,8]}$. And this highly depends on the physical parameters of the propagation problem. Actually, researchers have noticed HOKE in the context of ultrashort laser pulse propagation since $2000^{[9]}$. Some publications have discussed the sensitivity of the dominant physical effect to laser and medium condition ${ }^{[9,10]}$. As the interaction time of femtosecond pulse acting with air is very short, the avalanche ionization of air does not appear. This process is a complex nonlinear coupling process, where the optical Kerr effect, multiphoton absorption and ionization are the main physical mechanism of the beam filamentation. Other physical effects, including diffraction, group velocity dispersion and self-phase modulation, Raman-Kerr effect and self-steeping, have certain influence on the beam self-guidance propagation in the medium ${ }^{[11,12]}$. So the study on the influence of these physical effects in the process of propagation is one of the most attractive point.

Note that it is a tough task because of the difficulty to model the propagation of very large bandwidth pulse (>150 $\mathrm{nm}$ ) of ultrashort pulse duration (near Fourier limit) and the limits of validity of classical SVEA (slowly varying envelop approximation) hypothesis ${ }^{[3]}$. To relax this difficulty and to help to get a reliable and precise simulation tool, we have an interest in simulation of propagation in the context of non-converging or extremely loose focusing, that such situations can be used for initial test of the validity and precision of the developed models. The purpose of this research is to establish an accurate and reliable numerical simulation tool in our laboratories, so as to describe the propagation laws of femtosecond laser pulse with different pulse widths ( $\sim 10 \mathrm{fs}-500 \mathrm{fs}$ ) under the condition of atmospheric pressure and normal temperature. First of all, $2 \mathrm{D}+1$ full effect physical model was established on the basis of the nonlinear Schrödinger equation (NLSE). In this model, the nonlinear physical phenomena such as the diffraction, group velocity dispersion and high order dispersion effect, self-focusing, self-phase modulation, Raman Kerr effect, photoionization, plasma defocusing, plasma absorption and multi-photon absorption were considered. Then the physical process of 100 fs laser pulses propagating in air had been built using a numerical model, which revealed the filamentation characteristics of laser pulse and the nonlinear propagation laws of beam.

\section{Model and numerical method}

\subsection{D+1 propagation model based on NLSE}

To capture the physics of propagation of ultrashort laser pulses in air and more generally in gases, it is necessary to establish the propagation model which can describe the above nonlinear effects. In recent years, some international research groups have developed physical propagation models based on different unidirectional propagation equations
[14-16] to simulate the propagation process of femtosecond pulse, and capture the physical image of filamentation propagation using numerical experiments. The main idea is to establish the basic equation of pulse envelope evolution and then add a variety of different physical effects as source terms into the model.

The main idea of deriving wave propagation equation is to consider the linear polarization and nonlinear polarization process of electric field and magnetic field. Maxwell's equation is the basic equation to describe the spatial-time distribution of electric field and magnetic field, so the wave vector equation of propagation in medium can be derived from the Maxwell's equation. The current model is based on the NLSE wave propagation equation, which has almost become the standard equation in the research field of numerical simulation for propagation issues of ultrafast laser pulse ${ }^{[11]}$. More detailed nonlinear physical process has been simulated by adding different nonlinear source terms from the originally most simplified model only considering diffraction, optical Kerr effect and plasma absorption [11], to the extended model ${ }^{[6]}$ taking into account the nonlinear effects of plasma response process such as group velocity dispersion, and multi-photon ionization (MPI). In recent years, people researchers have begun to work on the influence of high-order Kerr effect on propagation ${ }^{[7,8]}$, and considered it as a dominant factor. In addition, the defocusing effect of the plasma is considered weakly, nearly having no effect on filamentation.

In the present work we developed a full model based on the extended model, which takes into account the linear effects such as diffraction and group velocity dispersion, as well as the nonlinear effects, e.g. optical Kerr effect, highorder Kerr effect, Raman Kerr effect, MPI and plasma defocusing. The third item on the right side of NLSE Eq. (1) is regarded as high-order Kerr effect, and the fourth item considers Raman Kerr effect. Equation (2) is the high-order nonlinear refraction coefficient, and Eq. (3) is plasma density equation, consistent with the extended model ${ }^{[6]}$. We recall here that NLSE equation (to be strictly valid) requires $\Delta k<<k_{0}$ and $\Delta \varepsilon<<\varepsilon$ (paraxial approximation). Expressed in the reference frame moving at the group velocity, the NLSE of a laser pulse with linearly polarized incident electric field $\varepsilon(r, t, z)$ along the propagation direction $z$ then read as:

$$
\begin{gathered}
\partial_{z} \varepsilon=i \frac{\Delta_{\perp}}{2 k_{0}} \varepsilon-i \frac{k^{\prime \prime}}{2} \partial_{t}^{2} \varepsilon+i \frac{k_{0}}{n_{0}} \Delta n_{\mathrm{ker}} \varepsilon+ \\
i k_{0} n_{2} n_{R} \int_{-\infty}^{t} R(t-\tau)|\varepsilon|^{2} \mathrm{~d} \tau-i \frac{k_{0}}{2 n_{0}^{2} \rho_{c}} \rho \varepsilon \\
-\frac{1}{2}\left(\sigma \rho \varepsilon+\beta^{(K)}|\varepsilon|^{2 K-2} \varepsilon\right) \\
\Delta n_{\mathrm{ker} r}=n_{2}\left(1-n_{R}\right)|\varepsilon|^{2}+n_{4}|\varepsilon|^{4}+n_{6}|\varepsilon|^{6}+n_{8}|\varepsilon|^{8} \\
\partial_{t} \rho=W(I)\left(\rho_{a t}-\rho\right)+\frac{\sigma}{U_{i}} \rho I-\alpha \rho^{2}
\end{gathered}
$$

where $t$ refers to the retarded time in the reference frame of the pulse $\left(t \rightarrow t-\frac{z}{v_{g}}\right.$ with $v_{g}=\left.\frac{\partial \omega}{\partial k}\right|_{\omega_{0}}$ )corresponding to the group velocity of the carrier envelope). The first two terms on the right-hand side of Eq.(1) are the linear effects, 
accounting for the diffraction and dispersion, the rest terms represent nonlinear effects of Kerr effect, Raman scattering effect, plasma defocusing effect and the multi-photon ionization effect. In the Raman scattering effect, it has the formulation of $R(t)=R_{0} \exp (-\Gamma t) \sin \left(\omega_{R} t\right)$, where $R_{0}=\left(\Gamma^{2}+\omega_{R}^{2}\right) / \omega_{R}$.

The Eq. (2) represent the HOKE terms, and the Eq. (3) represent the electric density equation where $I=|\varepsilon|^{2}$ and $W(I)=\sigma_{K}|\varepsilon|^{2 K}$.

The initial beam can be described by Eq. (4) which is regarded as a Gaussian pulse. The spatial distribution shape of laser pulse is a 2D+1 dimension model, where $r$ and $t$ represent the space domain and time domain respectively, and $z$ represents the pulse propagation distance.

$$
\varepsilon(r, t, z=0)=\varepsilon_{0} \exp \left(-\frac{r^{2}}{r_{0}^{2}}\right) \exp \left(-\frac{t^{2}}{t_{0}^{2}}\right) \exp \left(-i \frac{k_{0} r^{2}}{2 f}\right)
$$

\subsection{Numerical technique}

Obviously, it is necessary to search for the numerical solution of the partial differential equation based on NLSE, because it is impossible to obtain analytical solution for the propagation model based on NLSE equation. The Symmetric Split-Step Fourier Scheme (SSSF) has a higher efficiency to solve NLSE ${ }^{[15]}$, and the fast Fourier transform (FFT) is applied in the calculation. The computation efficiency of FFT is higher than the finite difference method. In the present physical process, linear effects such as diffraction and dispersion couple with nonlinear effects like self-focusing, multiphoton ionization in the light beam propagation. In general, we decompose the NLSE as

$$
\partial_{z} \varepsilon=(\hat{D}+\hat{N}) \cdot \varepsilon
$$

where $\hat{D}$ is an operator of the linear terms including diffraction and dispersion, and $\hat{N}$ is an operator of the nonlinear terms.

The split-step solution scheme is to treat the linear and nonlinear terms separately in the Fourier space over a step size of $h$. Over a half step all linear terms are computed (Fig. 1) while nonlinear terms are directly computed in the time domain. If the asymmetric scheme is used, Eq. (5) can be approximated as:

$$
\varepsilon(z+h, t) \approx \exp (h \hat{D}) \exp \left(\int_{z}^{z+h} \hat{N}(z) \mathrm{d} z^{\prime}\right) \varepsilon(z, t)
$$

and if the symmetric scheme (SSSF) is used, Eq. (5) can be approximated as:

$\varepsilon(z+h, t) \approx \exp \left(\frac{h}{2} \hat{D}\right) \exp \left(\int_{z}^{z+h} \hat{N}(z) \mathrm{d} z^{\prime}\right) \exp \left(\frac{h}{2} \hat{D}\right) \varepsilon(z, t)$

It can be seen from Baker-Hausdorff formula that the main error term of Eq. (6) is the second order $h^{2}$ item, and the main error term of Eq. (7) is the third order $h^{3}$ item. Therefore, the symmetrical scheme gets better accuracy.

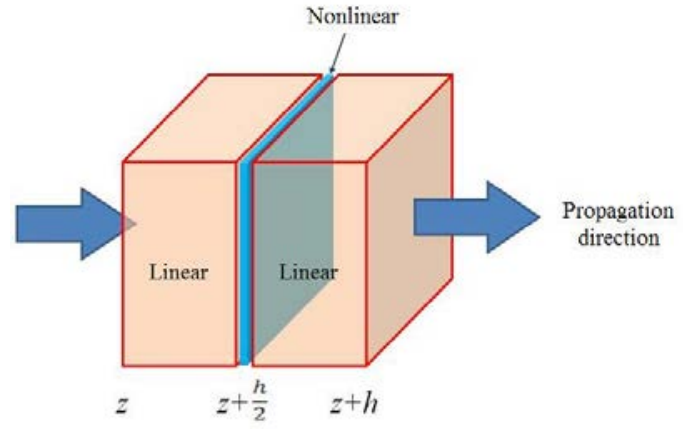

Fig.1 Schematic diagram of symmetrical split-step Fourier solving scheme.

It can be challenging to get numerical solution of NLSE on some technical issues, which including numerical format stability, calculation efficiency and the minimum calculation resolution (calculation step should be limited in a micron dimension), etc. All these issues are related to the definition of computing grid. Therefore, the initialization parameters of optical field can be defined as $E, \omega_{0}, \sigma_{r}, \sigma_{t}, f, C$.

The parameters of propagation medium including the refractive index of medium as well as the parameters of the plasma equation are also needed. As shown in Fig. 2, it is the definition method of the computing grid for the propagation of time domain and frequency domain space, where $2 \mathrm{D}+1$ grid is defined by six parameters $\left(\Delta r, N_{r}, \Delta t, N_{t}, \Delta z, N_{z}\right)$ :

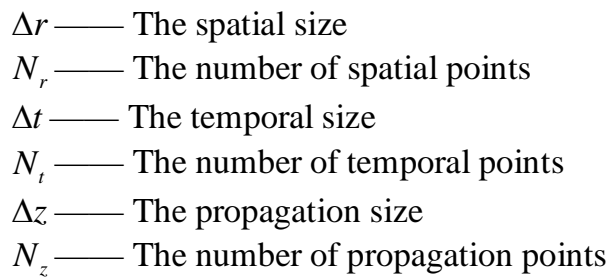

The calculation step is defined as

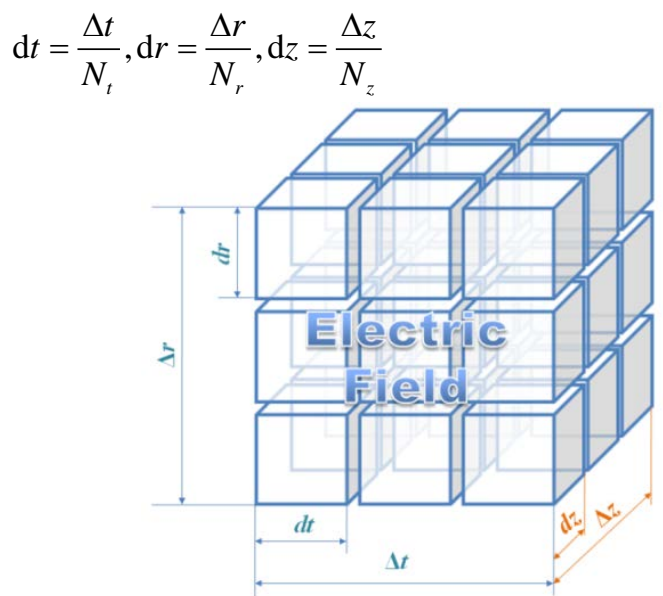

Fig.2 Definition of grid in transmission space

Because the physical effect of femtosecond laser filamentation experienced in $\mu \mathrm{m}$ and ns scales on the space and time domains, the simulation in the provision of such small step lengths not only needs the proper numerical format but consumes much computing time. According to 
literature and to our calculation results, dozens of hours in consumption is necessary in each simulation ${ }^{[6,8,16]}$. The code has run on high-performance computers such as DELL workstation.

\section{Results and discussion}

For a collimated Gaussian pulse with initial waist diameter of $1 \mathrm{~mm}$ and critical power $P_{\mathrm{cr}}=3.2 \mathrm{GW}$, the pulse duration is $100 \mathrm{fs}$ and the wavelength is $800 \mathrm{~nm}$, under one atmospheric pressure in air. Several conditions with input power near $P_{\text {cr }}$ have been simulated, and some calculation parameters are referred from literature [8].

Table.1 Calculation parameters

\begin{tabular}{|c|c|c|}
\hline $\begin{array}{l}\text { Symbol } \\
k_{0}\left(\mathrm{~cm}^{-1}\right)\end{array}$ & $\begin{array}{l}\text { Meaning } \\
\text { wave number }\end{array}$ & $\begin{array}{l}\text { Value } \\
7.85 \times 10^{4}\end{array}$ \\
\hline$k^{\prime \prime}\left(\mathrm{fs}^{2} \mathrm{~cm}^{-1}\right)$ & $\begin{array}{l}\text { second order dis- } \\
\text { persion coefficient }\end{array}$ & 0.2 \\
\hline$n_{0}$ & refractive index & 1.0003 \\
\hline$n_{2}\left(10^{-19} \mathrm{~cm}^{2} \mathrm{~W}^{-1}\right)$ & 3nd Kerr index & 3.2 \\
\hline$n_{4}\left(10^{-33} \mathrm{~cm}^{4} \mathrm{~W}^{-2}\right)$ & 5th Kerr index & -1.5 \\
\hline$n_{6}\left(10^{-46} \mathrm{~cm}^{6} \mathrm{~W}^{-3}\right)$ & 7th Kerr index & 2.1 \\
\hline$n_{8}\left(10^{-59} \mathrm{~cm}^{8} \mathrm{~W}^{-4}\right)$ & 9th Kerr index & -8.0 \\
\hline$n_{R}$ & $\begin{array}{l}\text { fraction of Raman } \\
\text { scattering }\end{array}$ & 0.5 \\
\hline$\Gamma(\mathrm{Hz})$ & $\begin{array}{l}\text { reciprocal of char- } \\
\text { acteristic time }\end{array}$ & $1.4 \times 10^{3}$ \\
\hline$\omega_{R}(\mathrm{~Hz})$ & $\begin{array}{l}\text { characteristic fre- } \\
\text { quency }\end{array}$ & $1.6 \times 10^{13}$ \\
\hline$\rho_{a t}\left(\mathrm{~m}^{-3}\right)$ & $\begin{array}{l}\text { neutral molecule } \\
\text { density of air un- } \\
\text { der } 1 \text { atm }\end{array}$ & $2.7 \times 10^{25}$ \\
\hline$\rho_{c}\left(\mathrm{~m}^{-3}\right)$ & $\begin{array}{l}\text { the critical density } \\
\text { of electron }\end{array}$ & $1.7 \times 10^{27}$ \\
\hline$\sigma\left(\mathrm{m}^{2}\right)$ & $\begin{array}{l}\text { inverse Brems- } \\
\text { strahlung cross } \\
\text { section }\end{array}$ & $5.6 \times 10^{-24}$ \\
\hline$U_{i}(\mathrm{eV})$ & $\begin{array}{l}\text { ionization poten- } \\
\text { tial }\end{array}$ & 12.063 \\
\hline K & $\begin{array}{l}\text { minimal number } \\
\text { of photons neces- } \\
\text { sary to ionize an } \\
\text { atom }\end{array}$ & 10 \\
\hline$\beta^{(K)}\left(\mathrm{m}^{-17} \mathrm{~W}^{-9}\right)$ & $\begin{array}{l}\text { multiphoton ioni- } \\
\text { zation coefficient }\end{array}$ & $1.27 \times 10^{-160}$ \\
\hline$\sigma_{K}\left(\mathrm{~s}^{-1} \mathrm{~m}^{2 K} \mathrm{~W}^{-K}\right)[21]$ & $\begin{array}{l}\text { cross section for } \mathrm{K} \\
\text { photons ionization }\end{array}$ & $\begin{array}{l}1.32776 \\
\times 10^{-168}\end{array}$ \\
\hline
\end{tabular}

During the calculation process, the different incident power $P_{\text {in }}$ is used to examine the features in the process of pulse propagation.

The evolution process of beam is showed as Fig.3 when the incident power $P_{\mathrm{in}}=0.3 P_{\mathrm{cr}}$. The Kerr effect does not appear and the diffraction divergence effect dominates in propagation because of the low incident power.

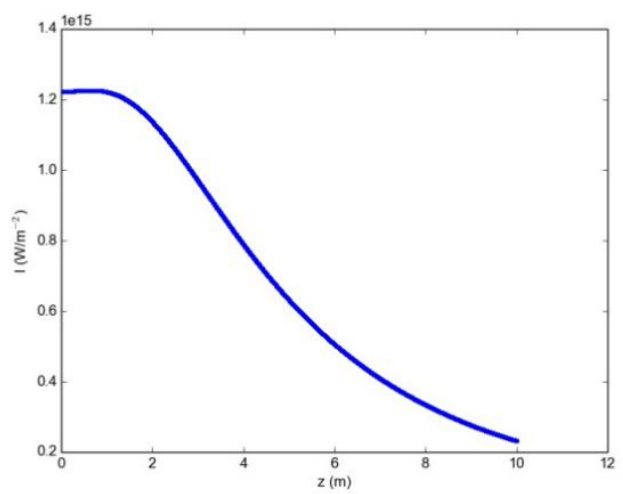

(a)The change of peak power density with propagation distance $z$

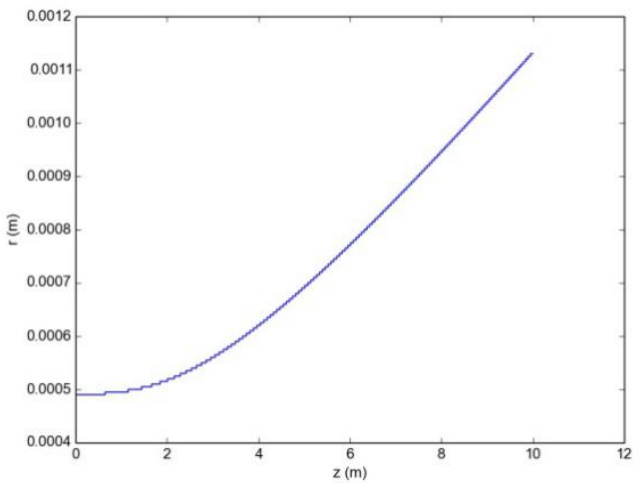

(b)The change of beam radius with propagation distance $z$

Fig.3 The evolution of laser pulse with propagation distance $\left(P_{\text {in }}=0.3 P_{\text {cr }}\right)$

Fig. 4 shows the evolution process of the beam when the incident power $P_{\mathrm{in}}=2 P_{\mathrm{cr}}$. The formation process of filamentation can be seen from the images. As shown in Fig.4 (a) and (b), Kerr effect induce self-focusing at $2.6 \mathrm{~m}$, and the beam size collapse which lead to the intensity within the pulse core becoming higher and higher. As the intensity reaches the ionization threshold, the generated plasma acts as a saturation mechanism, leading to the clamping of the pulse intensity. In addition, the collapse distance of the beam is also $2.6 \mathrm{~m}$ estimated by Marburger formula [17], which is consistent with the results of our numerical simulation. Subsequently, the beam begins to diverge. After propagation for $5 \mathrm{~m}$, the beam generates the self-focusing and defocusing cycle again. After $8 \mathrm{~m}$ of propagation, the beam is divergent because the energy cannot maintain the filamentation process. It turns out that the simulated results have a similar regularity compared to the existing literature $[6,11,18,19]$. Fig. 5 shows the evolution images of laser pulse power density at different times or propagation distances. It turns out that the results show a dominant effect of HOKE and a negligible role of plasma defocusing. Fig. 5 reflects the symmetry of HOKE terms whereas plasma defocusing would lead to an asymmetry between the leading and trailing part of the pulse. 


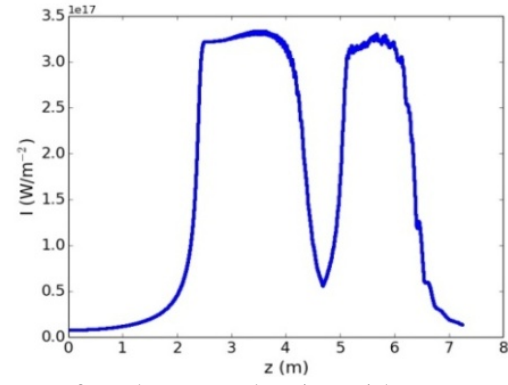

(a)The change of peak power density with propagation distance $z$

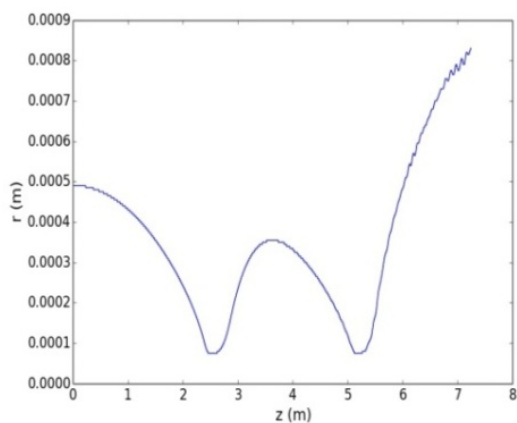

(b)The change of beam radius with propagation distance $z$

Fig.4 The change features of laser pulse filamentation with propagation distance $\left(P_{\mathrm{in}}=2 P_{\mathrm{cr}}\right)$
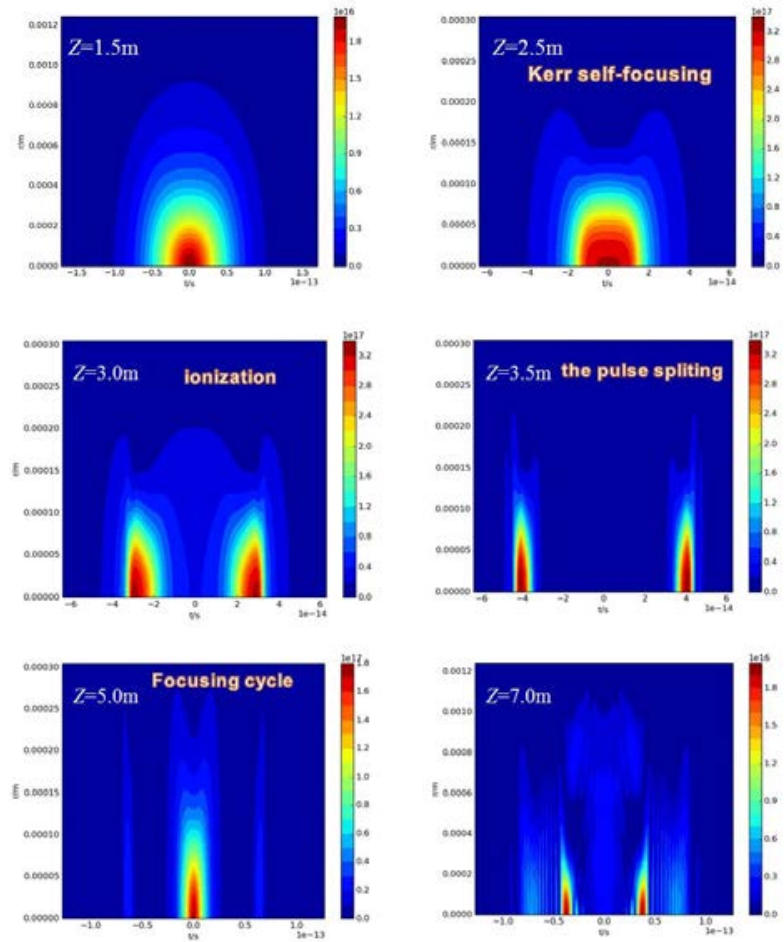

Fig.5 The evolution of laser pulse power density with the propagation distance

We have carried out numerical experiments for illustrating the competitive nonlinear effects by implementing the full model. We try to establish precise physical images with and without including corresponding terms of full model. Firstly, we switched off the HOKE effect to check the changes of propagation images (Fig.6). It reveals that in this test, the results without including HOKE have significant difference to the results of full mode. And then we switched off the plasma term in full model (Fig.7). The results without including plasma effect have similar with the results of full model. So now we take it as a preliminary conclusion that the HOKE take the dominated effect while the plasma defocusing take secondary effect, but not ignorable. In the following work, we will check the sensitivity of dominant effect between HOKE and plasma defocusing to laser's parameters and medium conditions.

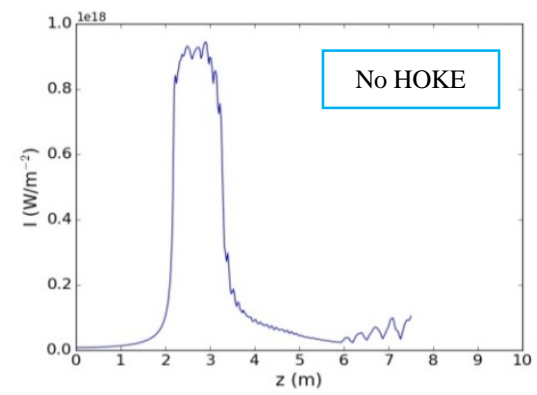

(a)The change of peak power density with propagation distance $z$

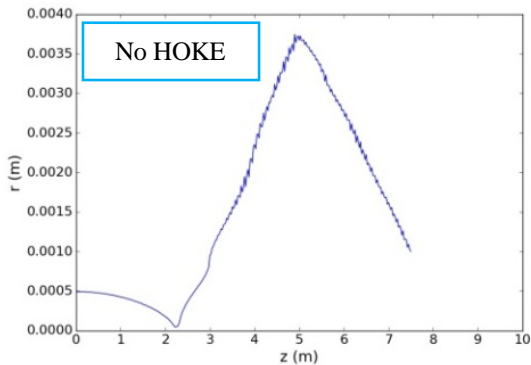

(b)The change of beam radius with propagation distance $z$

Fig.6 The change features of laser pulse filamentation with switching off HOKE in full model $\left(P_{\mathrm{in}}=2 P_{\mathrm{cr}}\right)$

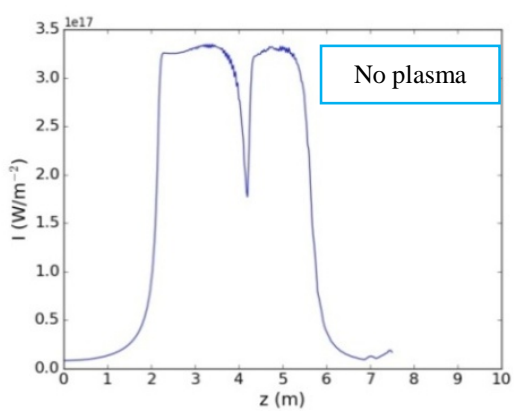

(a)The change of peak power density with propagation distance $z$

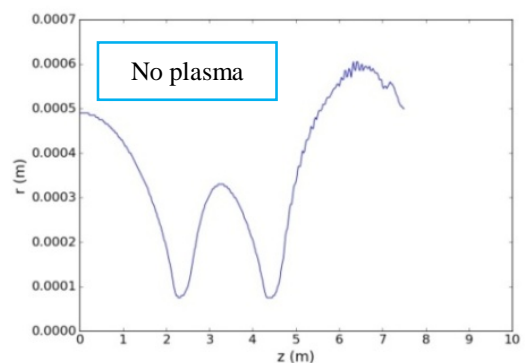

(b)The change of beam radius with propagation distance $z$

Fig.7 The change features of laser pulse filamentation with switching off plasma effects in full model $\left(P_{\mathrm{in}}=2 P_{\mathrm{cr}}\right)$ 
This model, however, fails for few cycle pulses when they are incorporated in our numerical tests. In fact, the parameters which are for instance described in the reference ${ }^{[3]}$, are here mentioned: NA 0.1 ( $f=50 \mathrm{~mm}$ and beam diameteris $3 \mathrm{~mm}$ ) for which simulations based on NLSE equation may failed. The simulation based this model can be not enough to explain the new regime of propagation in air in a nearly $10 \mathrm{fs}$ pulse combined with tightly focused geometry ${ }^{[2]}$ due to the limits of validity of classical SVEA. Nevertheless, assuming moderate restrictions on the pulse and the propagation medium, the Slowly Evolving Wave Approximation (SEWA) may be considered as an approximation in model propagation of few cycle pulses in medium ${ }^{[20]}$. In contrast to the SVEA, the SEWA does not imply a limitation of the pulse duration ${ }^{[12]}$.

\section{Conclusion}

We have established a numerical model and code for simulating the femtosecond laser pulse propagation in air, which are suitable for constructing the propagation and filamentation of narrow-band pulses in a Kerr medium. Several nonlinear physical effects are considered in this full model which include High-order Kerr effect (HOKE), Raman Kerr contribution, plasma absorption and defocusing, which can be implemented in laboratory for numerical construction adapted to weak focusing optics. This work has opened a door for further research on the beam propagation of ultrashort pulse width ( $<10 \mathrm{fs}$ ) combined with tightly focused geometry. It is worth noticing that, due to the required tiny space-time resolution, the numerical computation efficiency presents a great difficulty in the process of numerical simulation. So the next step is focused on developing the parallel computing technology to increase the calculation efficiency.

\section{References}

[1] N. Varkentina, N. Sanner, M. Lebugle, M. Sentis, O. Uteza: J. Appl. Phys. 114, (2013) 173105.

[2] C. Pasquier, P. Blandin, R. Clady, N. Sanner, M. Sentis, O. Uteza, Yu Li, Shen Yan Long: Optics Communications 355, (2015)230.
[3] B. Chimier, O. Uteza, N. Sanner, M. Sentis, T. Itina, P. Lassonde, F. Légaré, F. Vidal, J.-C. Kieffer: Phys. Rev. B. 84, (2011)094104.

[4] O. Uteza, N. Sanner, A. Brocas, B. Chimier, N. Varkentina, M. Sentis, P. Lassonde, F. Légaré, J.-C. Kieffer: Applied Physics A ,105, (2011)131.

[5] M. Lebugle, N. Sanner,, O. Uteza, M. Sentis: Appl. Phys. A, 114 (1), (2014)129.

[6] P.Bejot, Theoretical and experimental investigations of ultrashort laser filamentation in gases, doctoral thesis,Université De Genève

[7] P. Bejot, J. Kasparian: Optics Letters, 36, (2011)4812.

[8] X.X.Qi, et al: Optics Communications, 358, (2016) 126.

[9] Akozbek, et al:Phys. Rev. E, 61, 4550 (2000).

[10]A.Couairon, Phys. Rev. A, 68, 015801 (2003).

[11]A. Couairon, et al: Physics Reports . 441, (2007) 47.

[12]C. Brée, Nonlinear Optics in the Filamentation Regime, Springer Theses, (2012).

[13] S.L. Chin, S.A. Hosseini, W. Liu, Q. Luo, F.Theberge, N. Akozbek, A. Becker, V. Kandidov, O. Kosareva, H. Schroder: Can. J. Phys. 83, (2005)863.

[14]Moloney, J.V., Kolesik, M., Full vectorial, intense ultrashort pulse propagators: derivation and applications. In: Yamanouchi, K., Chin, S.L., Agostini, P., Ferrante, G. (Eds.), Progress in Ultrafast Intense Laser Science II, vol. 85. Springer, Berlin. (2007)

[15]A. Couairon, et al: The European Physical Journal Special Topics, 199, (2011)5.

[16]P. P. Kiran, et al: Physical Review A. 8, (2010) 013805.

[17]J.H.Marburger: Prog. Quant. Electr. 4, (1975)35.

[18]K. Lim, et al: Sci.. Rep. 4, (2014)7217.

[19]J.C. Diels, et al: Ultrashort laser pulse phenomena: Fundamentals, Techniques and Applications on a femtosecond time scale, Academic Press, San Diego, USA, 1996.

[20]Brabec and Krausz, Phys. Rev. Lett. 78, (1997) 3282.

[21] A. Couairon , et al: J. Opt. Soc. Am. B.19,(2002)

(Received: June 16, 2016, Accepted: January 5, 2017) 